\title{
Article
}

\section{Full-Length Transcriptome Sequencing and Comparative Transcriptome Analysis to Evaluate Drought and Salt Stress in Iris lactea var. chinensis}

\author{
Longjie $\mathrm{Ni}^{1,2,+}$, Zhiquan Wang ${ }^{1,+}$, Jinbo Guo ${ }^{1}$, Xiaoxiao Pei ${ }^{1}$, Liangqin Liu ${ }^{1}$, Huogen Li ${ }^{2}$, Haiyan Yuan ${ }^{1}$ \\ and Chunsun $\mathrm{Gu}$ 1,2,3,*
}

1 Institute of Botany, Jiangsu Province and Chinese Academy of Sciences, Nanjing 210014, China; LongJieNi@njfu.edu.cn (L.N.); wangzhiquan@cnbg.net (Z.W.); guojinbo@cnbg.net (J.G.); peixiaoxiao@cnbg.net (X.P.); liuliangqin@cnbg.net (L.L.); yuanhaiyan@cnbg.net (H.Y.)

2 College of Forest Sciences, Nanjing Forestry University, Nanjing 210037, China; hgli@njfu.edu.cn

3 Jiangsu Provincial Platform for Conservation and Utilization of Agricultural Germplasm, Nanjing 210014, China

* Correspondence: chunsungu@cnbg.net; Tel.: +86-25-84347051

+ These authors contributed equally to this work.

check for updates

Citation: Ni, L.; Wang, Z.; Guo, J.; Pei, X.; Liu, L.; Li, H.; Yuan, H.; Gu, C. Full-Length Transcriptome Sequencing and Comparative Transcriptome Analysis to Evaluate Drought and Salt Stress in Iris lactea var. chinensis. Genes 2021, 12, 434. https://doi.org/10.3390/genes 12030434

Academic Editor: Jong-Joo Cheong

Received: 9 February 2021

Accepted: 16 March 2021

Published: 18 March 2021

Publisher's Note: MDPI stays neutral with regard to jurisdictional claims in published maps and institutional affiliations.

Copyright: (c) 2021 by the authors. Licensee MDPI, Basel, Switzerland. This article is an open access article distributed under the terms and conditions of the Creative Commons Attribution (CC BY) license (https:// creativecommons.org/licenses/by/ $4.0 /)$.
Abstract: Iris lactea var. chinensis (I. lactea var. chinensis) is a perennial herb halophyte with salt and drought tolerance. In this study, full-length transcripts of I. lactea var. chinensis were sequenced using the PacBio RSII sequencing platform. Moreover, the transcriptome was investigated under $\mathrm{NaCl}$ or polyethylene glycol (PEG) stress. Approximately $30.89 \mathrm{G}$ subreads were generated and 31,195 unigenes were obtained by clustering the same isoforms by the PacBio RSII platform. A total of 15,466 differentially expressed genes (DEGs) were obtained under the two stresses using the Illumina platform. Among them, 9266 and 8390 DEGs were obtained under high concentrations of $\mathrm{NaCl}$ and PEG, respectively. In total, 3897 DEGs with the same expression pattern under the two stresses were obtained. The transcriptome expression profiles of I. lactea var. chinensis under $\mathrm{NaCl}$ or PEG stress obtained in this study may provide a resource for the same and different response mechanisms against different types of abiotic stress. Furthermore, the stress-related genes found in this study can provide data for future molecular breeding.

Keywords: Iris lactea var. chinensis; drought-stress tolerance; salt-stress tolerance; full-length transcripts; differentially expressed genes

\section{Introduction}

Plants are sessile organisms and are often affected by various abiotic stresses during their life cycle. These abiotic stresses include drought, salt damage, and toxic metals in soil, among which salt damage and drought stress are important factors limiting plant growth and productivity [1]. Moreover, with the increasingly frequent occurrence of extreme weather, the semi-arid and arid areas of the world continue to increase, forcing agriculture to gradually expand to semi-arid or arid areas. In addition, intensive agricultural irrigation will lead to changes in soil water balance in these areas and aggravate soil salinization [2]. Therefore, it is critical to increase plant resistance to drought and salt for agricultural production and the sustainable use of the environment. At the same time, the water and fertilizer consumption of stress-resistant plants will also be greatly reduced, thus reducing the cost and environmental burden.

Drought and salt can cause plants to have high permeability in the early stages of stress. At the same time, salt stress can cause an ionic imbalance. Over time, increased drought and salt stress will gradually generate oxidative stress and cause damage to cell components, such as membrane lipids, and proteins, and metabolic disorders caused by a series of complex secondary stress. Therefore, in response to drought and salt stress, plants have 
separate and common signal transduction mechanisms [3]. The most important feature of drought and salt stress is that it can promote the accumulation of the plant hormone abscisic acid (ABA), which leads to a series of adaptive responses in plants [4]. In addition, the increase of $\mathrm{Ca}^{2+}$ and reactive oxygen species (ROS) are key signal transduction components in the plant response to these two stresses [5]. When plants accumulate levels of $\mathrm{Ca}^{2+}$, ROS, and ABA, they will further regulate downstream drought and salt stress-related transcription factors (TFs) through signaling cascade reactions, thus further changing the transcription level in plants and improving stress resistance [6]. Current research on the mechanisms of drought and salt stress regulation has made great progress, for example, by finding the salt overly sensitive (SOS) pathway [7], ABA signal transduction pathway [8], and salt ion receptors glycosyl inositol phosphorylceramide (GIPC) sphingolipids [9]. However, more research about the underlying mechanisms of plant response to drought and salt stress is needed, especially in non-model plants.

I. lactea var. chinensis is a Chinese perennial herbaceous halophyte with wide adaptability and ornamental value. It is an important plant for ecological construction and the improvement of the soil environment [10]. Next-generation sequencing (NGS) technology has been used to reveal the regulatory mechanism of I. lactea var. chinensis under salt stress [11]. To our knowledge, however, there are no comparative studies on the common regulation mechanism of I. lactea var. chinensis under drought and salt stress. Moreover, due to the obvious disadvantages of second-generation sequencing, such as the short length of sequencing reads and unreliable assembly results, the accuracy of transcriptome abundance calculation is reduced to a large extent [12,13].

PacBio third-generation sequencing technology can effectively overcome problems associated with NGS. Longer read length, more uniform coverage, and more other advantages in building a complete transcriptome can be obtained by Pacbio, but at the same time, non-assembled long-read transcripts with a low error rate $(10 \%)$ can be generated by Pacbio, and the error rate can be overcome with correction of Illumina RNA-Seq [14]. Currently, these two sequencing technologies have been used to study different regulatory mechanisms in multiple species. For example, more transcriptional splice events have been found in rice by combining these two technologies [15], and they have both been used to reveal the complexity of ginsenoside biosynthesis and the transcriptome in Panax notoginseng [16]. Thus, for the first time, we constructed full-length reference transcriptomes of I. lactea var. chinensis under PEG and $\mathrm{NaCl}$ stress using isoform sequencing (ISO-seq sequencing) technology. According to the RNA-Seq data obtained by the Illumina sequencing platform, expression levels were compared and analyzed. The results revealed the changes of transcripts in I. lactea var. chinensis under $24 \mathrm{~h} \mathrm{PEG}$ and $\mathrm{NaCl}$ stress at the molecular level. These results will offer new data for the future study of the mechanism involved in the regulation of abiotic stress in I. lactea var. chinensis.

\section{Materials and Methods}

\subsection{Plant Materials, Growth Conditions, and Stress Treatment}

The plant materials of I. lactea var. chinensis, which were conserved in the Iris Resource Collection Garden of the Nanjing Sun Yat-Sen Memorial Botanical Garden (Nanjing, China), were used in this research. The seeds were germinated on moist filter papers for $24 \mathrm{~h}$ and then cultivated under a sterile condition in a greenhouse for three weeks $(16 \mathrm{~h} / 8 \mathrm{~h}$ light/dark; 65\% relative humidity). Afterward, the seedlings with similar tap root lengths were transferred into $50 \mathrm{~mL}$ centrifuge tubes and then cultured in half-strength Murashige and Skoog (1/2 MS) nutrient solution at pH 5.8 for one week. The solution was refreshed every two days during the seedling growth. Then the seedlings were set into $1 / 2 \mathrm{MS}$ containing $1.5 \% \mathrm{NaCl}$ or $30 \%$ PEG for $24 \mathrm{~h}$, and the control seedlings were still cultured in standard 1/2 MS solution. The control and treatments were repeated three times with a total of nine biological replicates, and all samples (leaves and roots (approximately $1.5 \mathrm{~cm}$ in length)) were sampled at the same time for sequencing analysis; the samples were frozen in liquid nitrogen and deposited at $-80^{\circ} \mathrm{C}$. 


\subsection{Iso-Seq Library RNA Preparation, Sequencing, and Analysis}

The RNAiso reagent (TaKaRa Biotech Co., Dalian, China) was used to extract total RNA from each sample. A 1\% agarose gel was used to monitor RNA degradation and contamination. Nanodrop 2000 spectrophotometer (Thermo Fisher Scientific Inc., Walthman, MA, USA) and Agilent 2100 Bioanalyzer (Agilent Technologies, Palo Alto, CA, USA) were used to monitor protein contamination (A260/A280 ratio) and reagent contamination (A260/A230 ratio) to validate RNA accuracy. The total RNA with ratios of A260/A280 and A260/A230 between 1.8 and 2.0 were chosen for subsequent analysis. The Qubit 2.0 fluorometer (Invitrogen, Carlsbad, CA, USA) was used to accurately quantify the RNA concentration of all samples.

The concentration of RNA of each sample was greater than $300 \mathrm{ng} / \mathrm{ul}$, and the total amount of the RNA used for cDNA library was greater than $5 \mathrm{ug}$. The SMARTer ${ }^{\circledR}$ PCR cDNA Synthesis Kit (Clontech, Mountain View, CA, USA) was used to synthesize the first-strand cDNA, and then second-strand cDNA was synthesized by large-scale PCR. A Kapa Hifi PCR package (KAPA Biosystems, Boston, MA, USA) was used for PCR amplification and cDNA synthesis. Bluepippin was used to screen fragments (optional size $>4 \mathrm{~kb}$ ) and a SMRTBell template Planning kit 1.0 (Clontech, CA, USA) was used to build the SMRTBell library for damage and final repair. Finally, the stem-loop sequencing adapter was attached to both ends of the DNA fragments and exonuclease was used to delete the failed fragments. After the library was quantified, the library prototype enzyme complex was sequenced on a PacBio sequel system. The PacBio RSII tool (Pacific Bioscience Inc., Menlo Park, CA, USA) was used to sequence the cDNA library and then process polymerase readings to extract low-quality and short-read sequences. High-quality full-length sequences were obtained by grouping, clustering, and correction. SMRTlink 5.1 (http:/ / www.pacb.com/products-and-services/analytical-sofware/smrt-analysis/, accessed on 1 August 2017) software was carried out for processing the above data, and the parameters are set as follows: min_length 300, max_drop_fraction 0.8, no_polish TRUE, min_zscore -9999, min_passes 1, min_predicted_accuracy 0.8, max_length 15,000.

CD-HIT (version: 4.6.7) was used to cluster the corrected transcript sequences according to the $95 \%$ similarity between the sequences, and the parameters are set as follows: -c 0.85, -T 6, -G 0, -aL 0.00, -aS 0.99, -AS 30 [17]. The core conserved gene set of terrestrial plants, namely, Eukaryota (version: V1, number of BUSCOs: 429), and BUSCO (version: 3.0.2) were used to evaluate the completeness of the full-length transcriptome sequences [18]. The TransDecoder program was used to estimate the ORF of the transcripts (version: 5.5.0; parameter: $-\mathrm{m}$ 50). The identification parameters were as follows: (1) open reading frame (ORF) length is greater than $300 \mathrm{bp}$ and (2) logarithm value of the probability function is greater than 0 .

All full-length transcripts were annotated using seven online libraries, including the NCBI non-redundant protein (NR) database, the Cluster of Orthologous Groups of proteins (COG), UniProt Knowledgebase (UniProt), the Kyoto Encyclopedia of Genes and Genomes (KEGG) database, the EuKaryotic Orthologous Groups (KOG) database, the Gene Ontology (GO) database, and the Protein Family (Pfam) database. The first five annotation databases were performed using DIAMOND (version: 0.8.36) with an E-value threshold of $1.0 \times 10^{-5}$. HMMER 3.1 package was used for Pfam database annotation. Blast2GO v5.1 (http:/ / www.blast2go.com, accessed on 1 May 2018) and a script were used for GO annotation.

\subsection{RNA-Seq Library RNA Preparation, Sequencing, and Analysis}

The amount of RNA of each sample was $1.5 \mathrm{ug}$ for RNA-Seq library construction. NEBNext ${ }^{\circledR}$ Ultra $^{\mathrm{TM}}$ RNA Library Prep Kit for Illumina ${ }^{\circledR}$ (NEB, USA) was used to generate an RNA-Seq library following the manufacturer's protocol. AMPure XP system (Beckman Coulter, Beverly, CA, USA) was used to select cDNA fragments of preferentially 250 300 bp in length and purify PCR products. Illumina Hiseq2000 (Illumina Inc., San Diego, CA, USA) was used for library sequencing and generating paired-end reads. 
A full-length non-chimeric transcript was used as a reference sequence to obtain isoform after removing redundancy with CD-HIT, and Bowtie2 (version 2.3.4; Parameter: Mismatch 0) software was used to compare the second-generation high-throughput sequencing data with the above reference sequence. RSEM (version: 1.3.1) was used to obtain the fragments per kilobase of exon model per million mapped fragments (FPKM) based on the read count for each gene in each sample, and the FPKM value was converted to transcript per million (TPM) value to analyze the expression level [19].

\subsection{DEGs Analysis}

The edgeR was used to screen DEGs based on read counts, FDR $<0.05$ and $\mid \log _{2}$ (Foldchange) $\mid>1$ were set as the threshold [20]. Then DEGs was also assessed via the DEseq2 R package, qvalue $<0.05$ and $\mid \log _{2}$ (Foldchange) $\mid>1$ were set as the threshold [21,22]. Finally, the DEGs, which were screened by both of the two soft wares, were used for subsequent analysis.

For function annotation, we used the GOseq R packages based on Walleniusnoncentral hypergeometric distribution to perform GO enrichment analysis [23]. The KEGG enrichment analysis was carried out using KOBAS software [24], Venn diagrams and heat maps were drawn by TBTools (version: 1.007) [25], and the gene expression map was drawn by MapMan (version: 3.5.1) [26]. Coexpression networks were constructed and used with the WGCNA R packages (version: 1.68) [27], and visualization was performed with Cytoscape (version 1.7) [28]. The Cytoscape plugin cytoHubba was used to identify hub genes and rank them according to the maximal clique centrality (MCC) scores [29].

\section{Results}

\subsection{Single-Molecule Real-Time (SMRT) Sequencing, Data Processing, Annotation, and Coding} Sequence (CDS) Prediction

Total RNA was extracted from the root, stem, and leaf tissues of nine samples, including $24 \mathrm{~h}$ of $\mathrm{NaCl}$ treatment, $24 \mathrm{~h}$ of PEG treatment, and controls without any treatment. Almost equal amounts of high-quality RNA were mixed to generate an informative reference transcript database. Full-length transcriptome data were obtained by the PacBio Sequel platform, and approximately $30.89 \mathrm{G}$ subreads $(258,615$ circular consensus sequencing (CCS)) were generated. After filtering out incomplete CGs, 168,924 full-length nonchimeric read (FLNC) sequences with complete $5^{\prime}-3^{\prime}$ ends were obtained. After clustering redundant sequences, 99,483 consensus sequences were obtained, and then 62,717 isoforms were obtained by CD-HIT software (Figure 1a). Finally, 31,195 unigenes were obtained by clustering the same isoforms (Figure $1 \mathrm{~b}$ ), and the length of these sequences ranged from 161 to $6386 \mathrm{bp}$. The average size was $1564 \mathrm{bp}$, and the length of ExN50 was $1115 \mathrm{bp}$. A total of $23,244(74.51 \%)$ transcripts were over $1000 \mathrm{bp}$ in length. Based on benchmarking universal single-copy ortholog (BUSCO) analysis, approximately 307 (72\%) of the 429 expected embryophyte genes were identified as complete (Supplementary Materials Table S1). A total of 30,295 CDS sequences were predicted by TransDecoder software, among which $327(1.07 \%)$ CDS sequences were longer than $3000 \mathrm{bp}, 24,167$ (79.75\%) were between $400 \mathrm{bp}$ and $3000 \mathrm{bp}$, and $5801(19.15 \%)$ were less than $400 \mathrm{bp}$ (Table 1), the remaining 900 genes without CDS may be pseudogenes and lncRNAs [30]. 


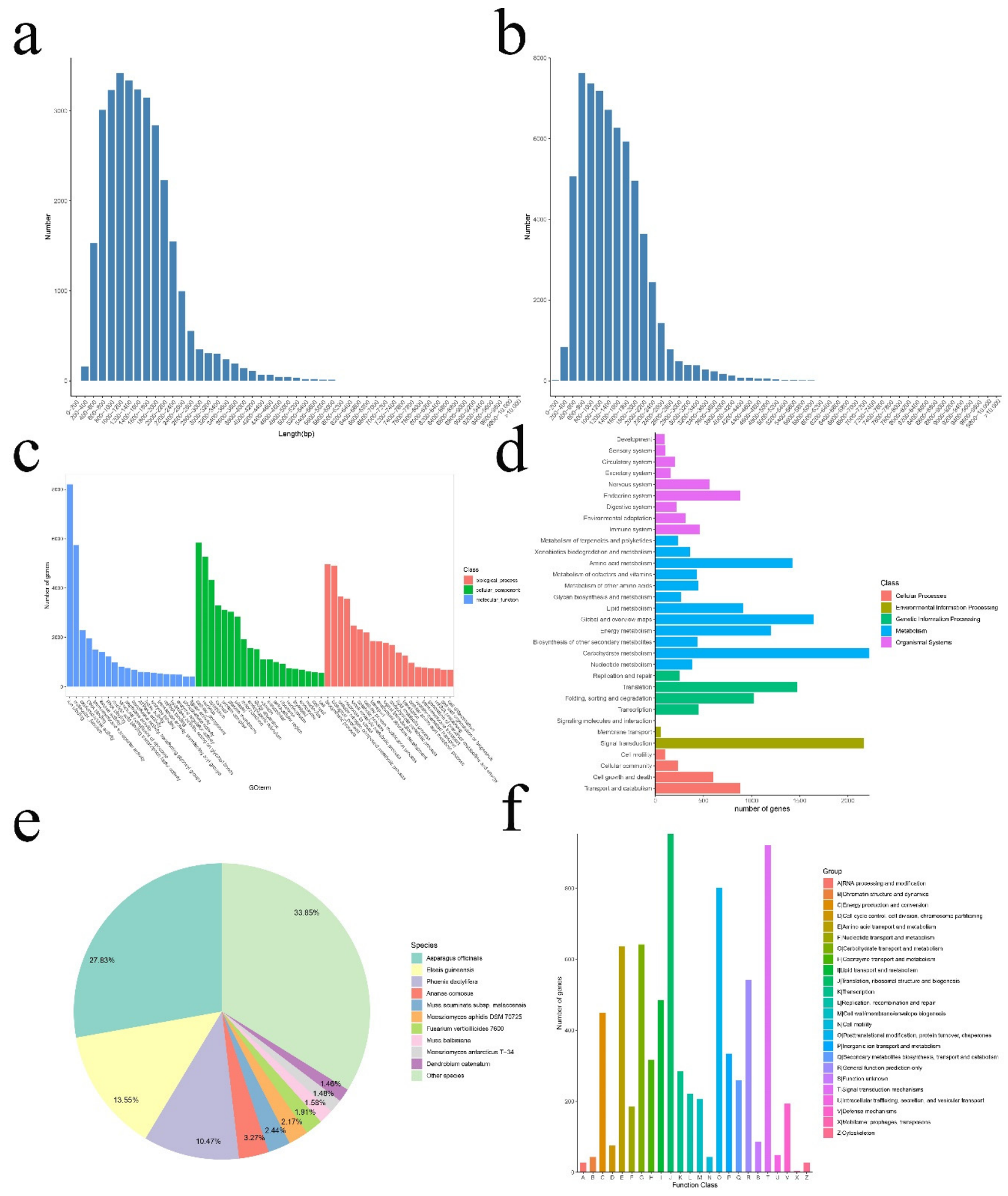

Figure 1. Iso-Seq sequencing, assembly, and annotation of I. lactea var. chinensis. (a) Length distribution of the isoform. (b) Length distribution of the full-length transcripts. (c) Gene Ontology (GO) classification of the assembled full-length transcripts. (d) Kyoto Encyclopedia of Genes and Genomes (KEGG) annotation of the assembled full-length transcripts. (e) The distribution of homologous species annotated in the NCBI non-redundant protein (NR) database. (f) Cluster of Orthologous Groups of proteins (COG) classification of the assembled full-length transcripts. 
Table 1. Summary for the transcriptome data of I. lactea var. chinensis using PacBio.

\begin{tabular}{cc}
\hline & Pacbio \\
\hline Subreads base (G) & 30.89 \\
Subreads number & $21,487,251$ \\
Average subreads length & 1438 \\
CCS & 258,615 \\
5'-primer $^{\prime}$-primer & 249,012 \\
Poly-A & 249,046 \\
Flnc & 232,150 \\
Average flnc read length & 168,924 \\
Consensus reads & 1304 \\
Number of isoform & 99,483 \\
Number of unigene & 62,717 \\
Mean_length & 31,195 \\
Min_length & 1564 \\
Max_length & 161 \\
ExN50 (consensus) & 6386 \\
ExN90 (consensus) & 1115 \\
Number of Genes & 1693 \\
Number of CDS & 31,195 \\
\hline
\end{tabular}

In this study, 31,195 unigenes were annotated using seven databases. The number of annotated unigenes in the seven databases ranged from 8633 (27.67\%, KEGG pathways) to $28,063(89.96 \%, N R)$, and $28,419(91.10 \%)$ unigenes were annotated in at least one database (Table S2). In addition, 21,697 unigenes were annotated to the GO database (Figure 1c) and 12,556 unigenes were annotated to the KEGG database (Figure 1d). A large number of genes were annotated with COG to the RNA processing and modification, followed by signal transduction mechanisms, defense mechanisms, and energy production andconversion (Figure 1f).

\subsection{Illumina Sequencing Data Analysis and DEGs Screening}

Using the Illumina platform, nine cDNA libraries (three replicates per process) were sequenced. A total of 431,683,966 reads were generated, with an average of $47,964,885$ reads per library. Then, all reads were mapped to the full-length transcriptome database of I. lactea var. chinensis through the Bowtie2 software. The final alignment was $215,841,983$ reads, and the average alignment was $23,250(74.53 \%)$ unigenes. A total of 62,718 transcripts were identified in the nine sample libraries. Each library contained more than 20,000 genes. The average mapping rate of all libraries of RNA-seq in this study was $75.9 \%$, suggesting that the library of PacBio had a high degree of integrity (Table S3).

Based on the reads counts, DEGs were assessed with the DESeq2 program. A total of 15,466 DEGs were found to participate in response to the two stresses. Among them, compared with the CK, 9266 (5148 up-regulated, 4118 down-regulated) and 8390 (4827 upregulated, 3563 down-regulated) DEGs were obtained under $\mathrm{NaCl}$ and PEG treatment, respectively (Figure 2c-f). 
a

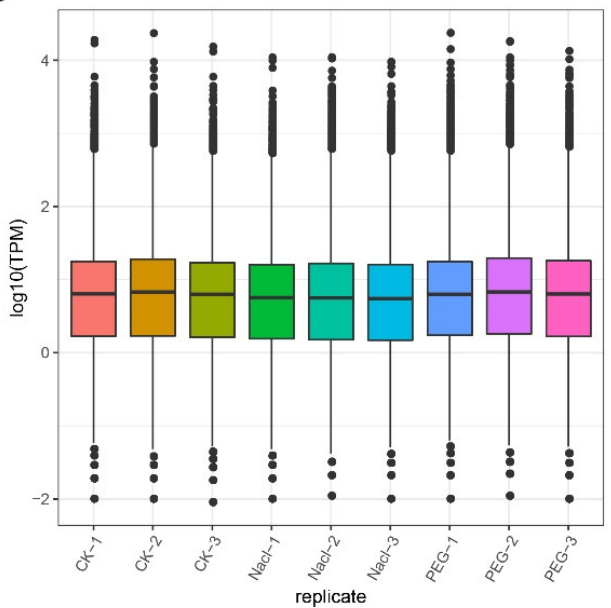

c

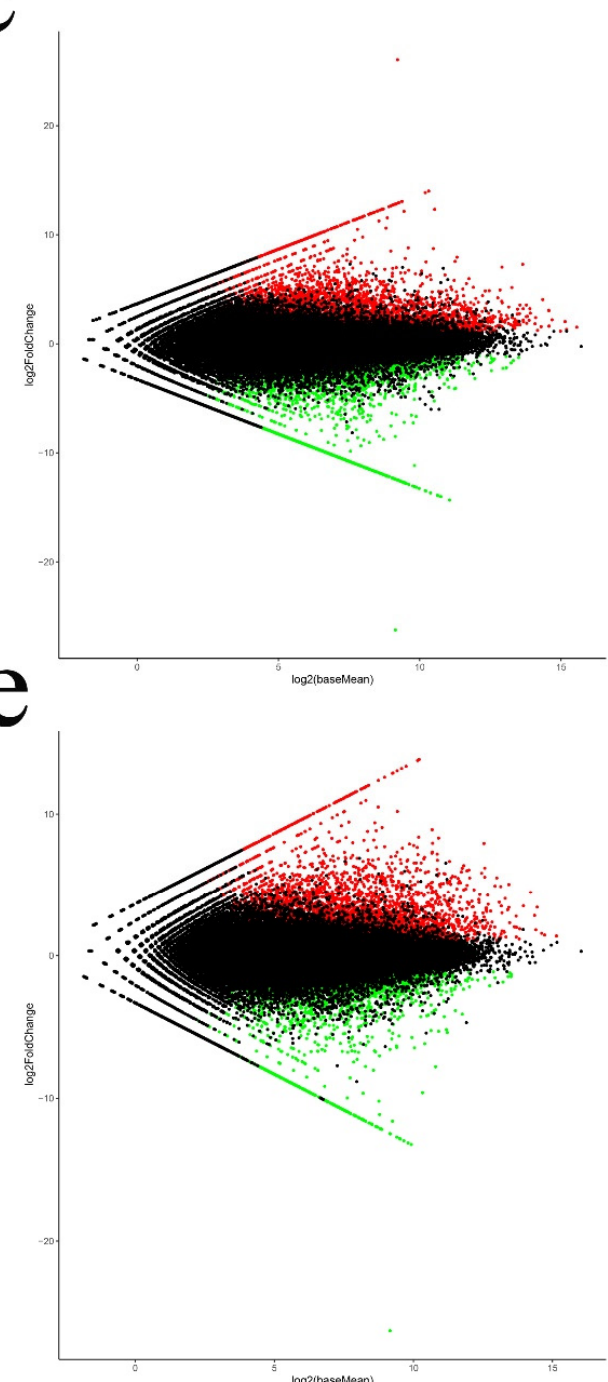

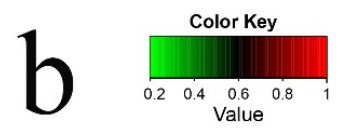
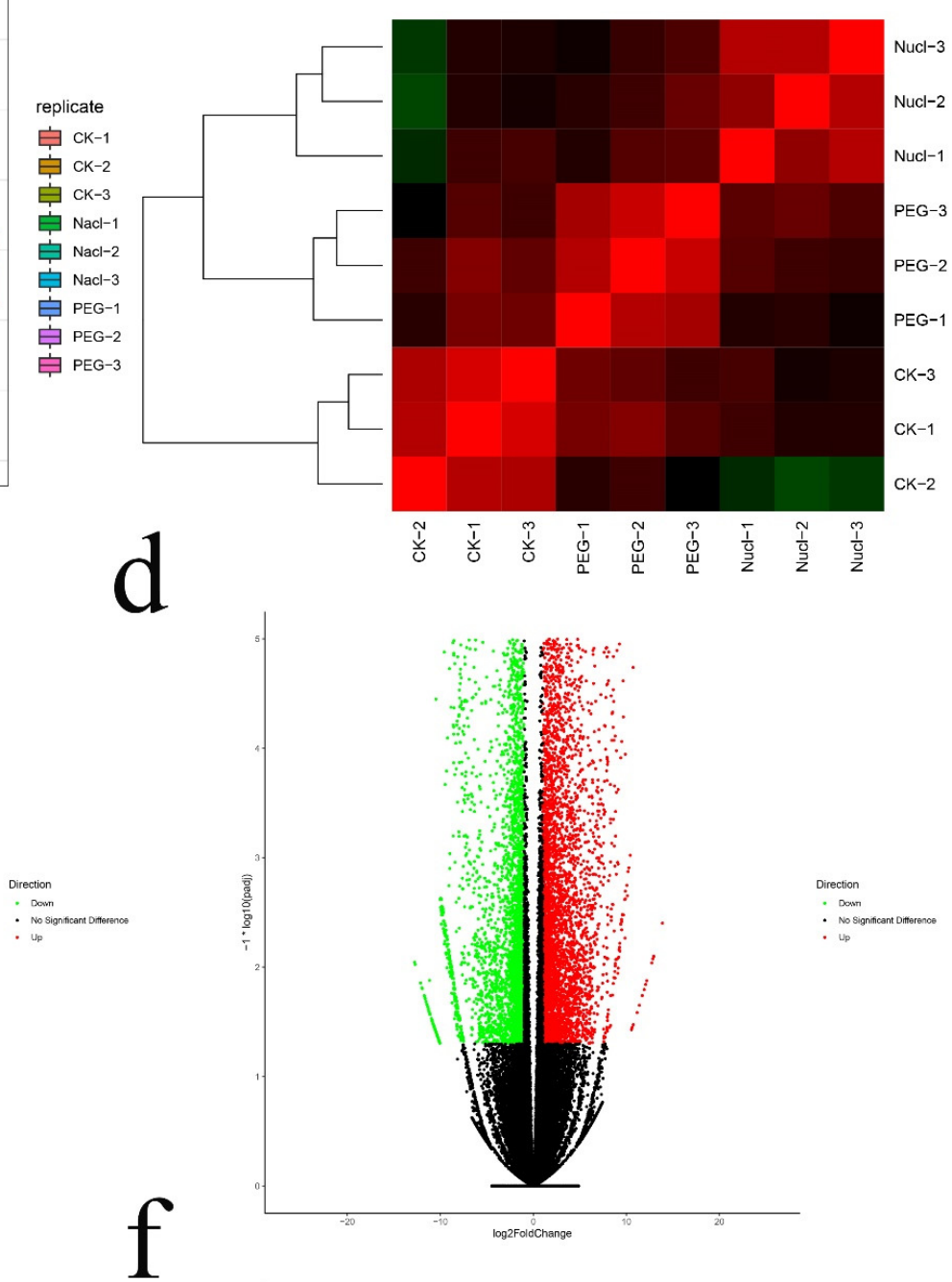

Direction
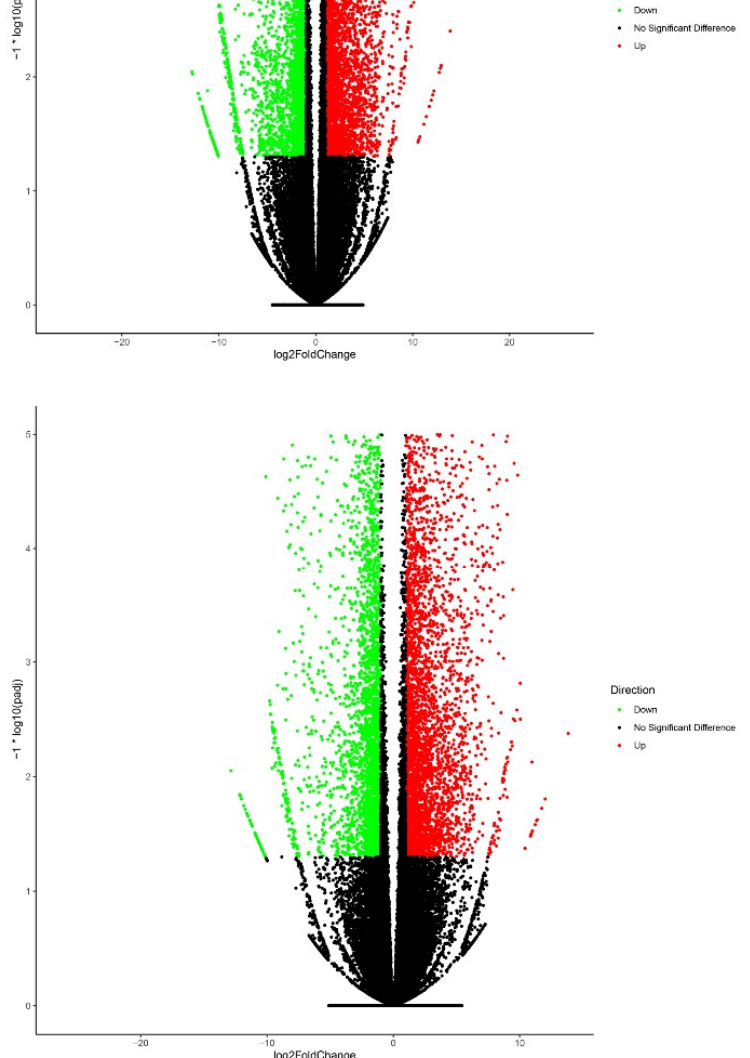

Figure 2. (a) The transcript per million (TPM) box plots. (b) Clustering analysis diagram between samples. (c) CK_VS_NaCl differentially expressed genes (DEGs) M-versus-A (MA) plot. (d) CK_VS_NaCl DEGs volcanic plot. (e) CK_VS_PEG DEGs MA plot. (f) CK_VS_PEG DEGs volcanic plot. 


\subsection{Analysis of Stress Regulation Networks}

To screen the key regulatory genes under the two stresses further, weighted gene coexpression network analyses (WGCNA) of all DEGs were performed, and DEGs were divided into 12 different hierarchical clustering modules. Different colors (blue, darkgreen, grey, darkgrey, darkred, green, lightgreen, paleturquoise, red, royalblue, steelblue, and white) were used to represent different modules, and GO enrichment analysis was carried out for different modules (Figure 3a,b).

\section{a}

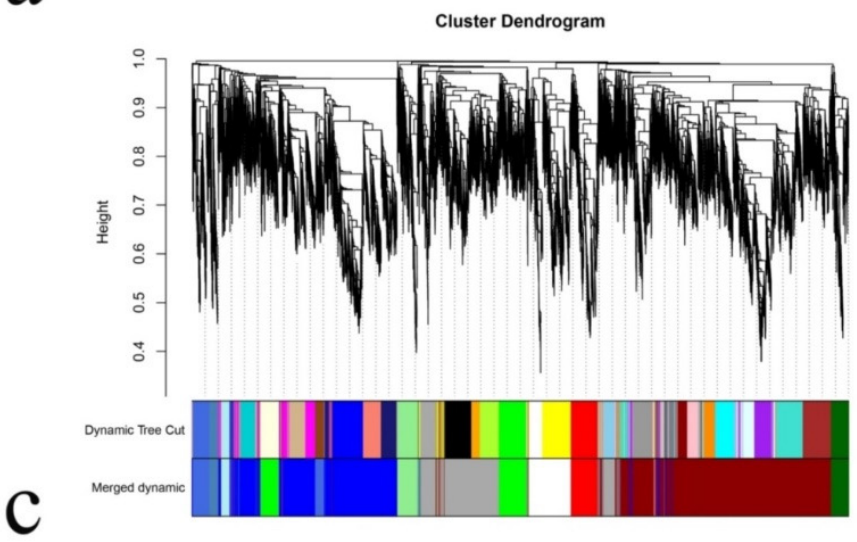

b
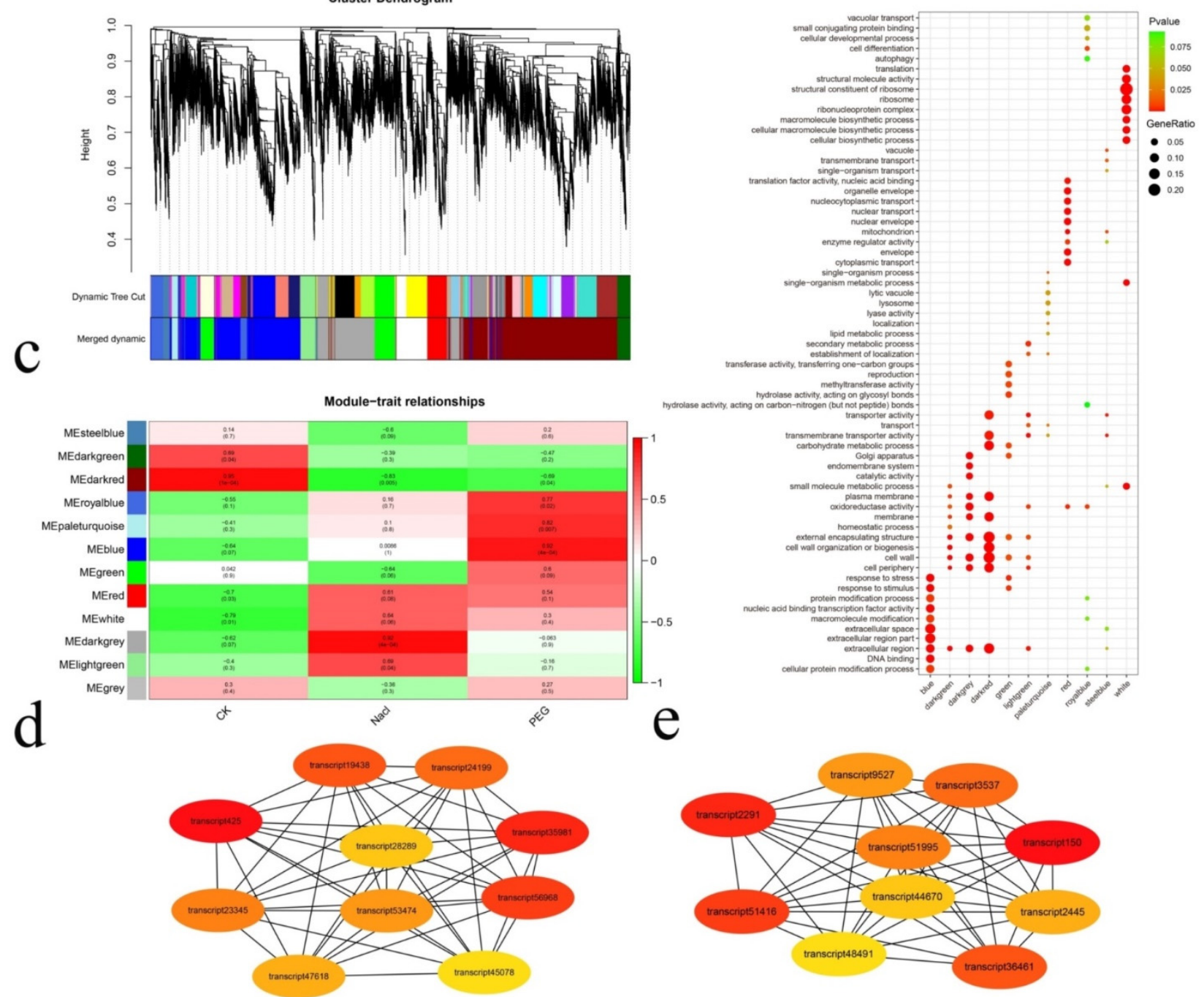

Figure 3. Construction of the gene co-expression network through weighted gene co-expression network analyses (WGCNA). (a) Gene dendrogram obtained by hierarchical clustering with the module color. A total of 12 distinct modules were identified. (b) Scatterplot of GO enrichment for different modules. The size and color of the dots represent the gene ratio and the range of the P-value, respectively. (c) Relationships of modules and different samples. Each row in the table corresponds to a module, and each column corresponds to a sample. (d) The 10 hub genes under $\mathrm{NaCl}$ stress. (e) The 10 hub genes under polyethylene glycol (PEG) stress.

The largest module, darkred, contained 5053 DEGs, most of which were enriched in the membrane, transmembrane transporter activity, plasma membrane, and other GO terms related to the transporter, and showed the strongest positive relationship with the control group. The second module, blue, contained 3447 DEGs. Most of the genes in this module were enriched in response to stimuli, protein modification process, and other GO 
terms closely related to metabolism, and showed the strongest positive relationship with PEG stress. The third module, darkgrey, contained 2192 DEGs. Most of the genes in this module were enriched in catalytic activity, cell wall, membrane, and other GO terms related to cell structure, and showed the strongest positive relationship with $\mathrm{NaCl}$ stress (Figure 3c).

Cytoscape Plugin Cytohubba was used to selected 10 DEGs, as the key genes, from the darkgrey and blue modules and were annotated with NCBI blast (Figure 3d,e). The key genes under salt stress were annotated as calcium permanent channel 1 (OSCA1), probable protein phosphatase $2 \mathrm{C}(P P 2 C)$, mitogen-activated protein kinase (MAPK), calciumdependent protein kinase 19 (CDPK), abscisic acid receptor PYL10 (PYL10), dihydroflavonol 4-reductase (DFRA), chalcone synthase 1 (CHS1), ATP-citrate synthase $\beta$ chain protein 1 (ACLB1), calcium-dependent protein kinase 5 (CDPK5), and acetyl-CoA carboxylase $1(A C C 1)$ (Table S4), and the key genes under drought stress were annotated as phosphoenolpyruvate carboxylase kinase 1 (PPCK1), $P P 2 C$, succinate, probable WRKY transcription factor 15 (WRKY15), calcineurin B-like (CBL) interacting protein kinase (CIPK8), stemspecific protein (TSJT1), glutathione S-transferase (GST), serine/threonine-protein kinase (STY17), and translationally controlled tumor protein homolog (Table S5).

\subsection{Analysis of Comparative Transcriptome}

A total of 15,466 DEGs were found participating in response to the two stresses (Figure 4a). All the DEGs were divided into four categories according to their expression patterns under different stresses. Among them, those that were upregulated under $\mathrm{NaCl}$ and PEG stress were divided into categories I and III, and those that were downregulated under $\mathrm{NaCl}$ and PEG stress were divided into categories II and IV. GO enrichment analysis showed that the DEGs under $\mathrm{NaCl}$ and PEG stress have similar functions. Among them, transporter activity, transmembrane transporter activity, cell wall, cell periphery, and membrane were significantly enriched under the two stresses. However, only type I, type II, and type IV genes were significantly enriched in these terms, and not type III. This suggested that the same genes may play different roles in response to these two stresses. In addition, transmembrane transport, small molecular metabolic processes, and oxidoreductase activity were only enriched in type I and type III, indicating that these terms may play a positive regulatory role in response to the two stresses. On the other hand, carbon metabolic processes, plasma membrane, and vacuum were only enriched in type II and type IV, indicating that these terms may play a negative regulatory role in response to the two stresses. In addition, transport, lipid metabolic process, peptidase activity, and catalytic activity were only enriched under $\mathrm{NaCl}$ stress, whereas DNA binding, Golgi attachments, nuclear acid binding transcription factor activity, and response to stimulus were only enriched in PEG stress, indicating that I. lactea var. chinensis had different defense mechanisms in response to the two stresses (Figure 4 b).

To explore the common defense mechanism of I. lactea var. chinensis in response to the two stresses further, we analyzed DEGs with the same expression pattern under the two stresses separately. In this study, 3863 DEGs had the same expression pattern under the two stresses, and the remaining 34 DEGs had different expression patterns under the two stresses (Figure 4a). Among them, 2508 DEGs were upregulated and 1355 DEGs were downregulated. GO enrichment analysis showed that the two types of DEGs were enriched in five GO terms, namely transporter activity, transmembrane transporter activity, external covering structure, cell wall, and catabolic process, which were closely related to stress response, indicating that these GO terms may play important roles in response to stress. In addition, upregulated DEGs were specifically enriched to nine GO terms, including oxidoreductase activity, response to stress, single-organization transport, and transmembrane transport, indicating that these terms may play positive regulatory roles in response to stress, whereas down-regulated DEGs were specifically enriched to seven GO terms, including the plasma membrane, membrane, cell periphery, and carbohydrate metabolic process, suggesting that these terms might play a negative regulatory role in response to stress (Figure 4c). KEGG enrichment analysis was used to understand the complex 
biological behavior. Overall, 3863 DEGs with the same expression pattern were enriched in 17 pathways, including amino acid metabolism, carbon hydrate metabolism, signaling, and cellular processes, ion channels, transporters, and other pathways related to metabolism and ion transport (Figure 4d). This suggested that the defense of I. lactea var. chinensis in response to these two stresses was through metabolism and ion transport pathways.

a

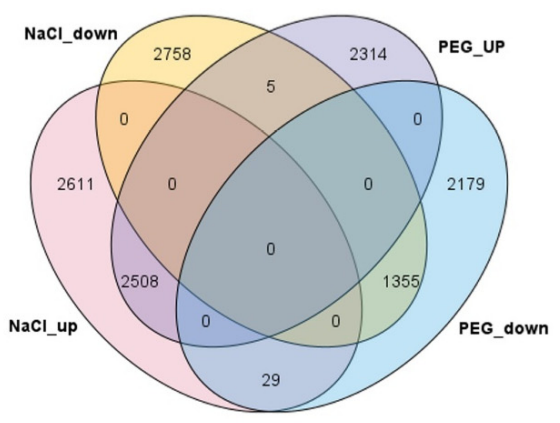

C

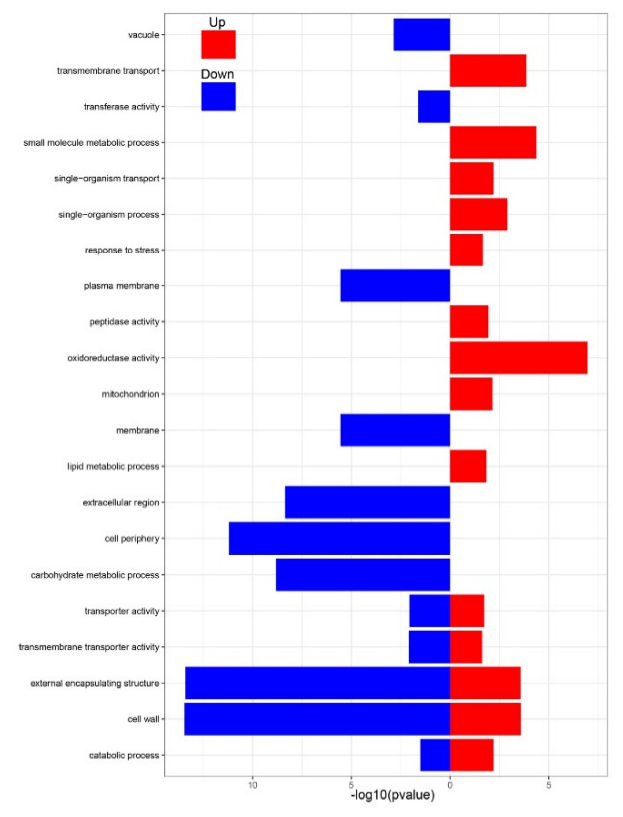

b
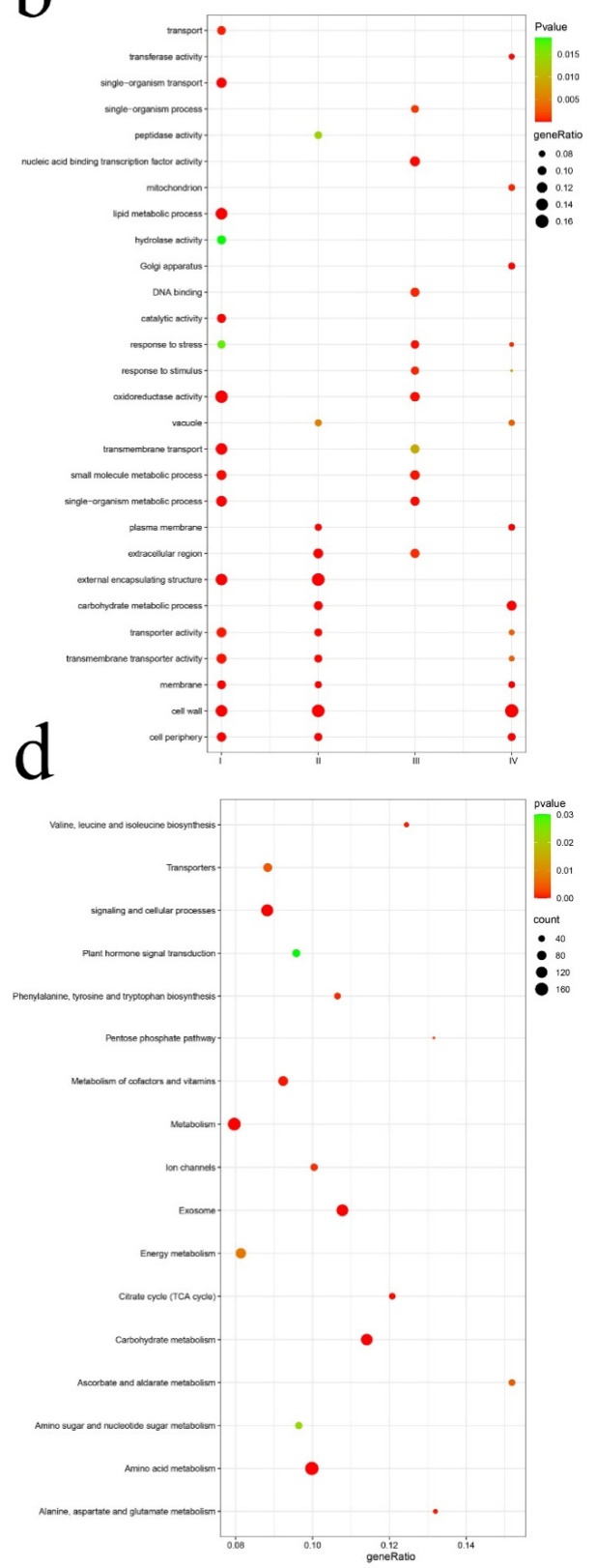

Figure 4. Comparative analysis of the I. lactea var. chinensis transcriptomes. (a) Venn diagram of all DEGs. (b) GO enrichment of DEGs that were differentially expressed between control and treatments, the size and color of the dots represent the gene ratio and the range of the $p$-value, respectively. (c) GO enrichment of DEGs that had the same expression between $\mathrm{NaCl}$ and PEG stress. The degree of GO enrichment is represented by the $-\log _{10}$ ( $p$-value). (d) Scatterplot of enriched KEGG pathways for DEGs that had the same expression under $\mathrm{NaCl}$ and PEG stress. The rich factor is the ratio of the DEG number to the total gene number in a certain pathway. The size and color of the dots represent the gene number and the range of the $\log _{10}$ ( $q$-value), respectively. 
Osmotic stress is the core of the plant response to drought and salt stress; hence, we mapped the related DEGs to the osmotic stress regulatory network (Figure 5).

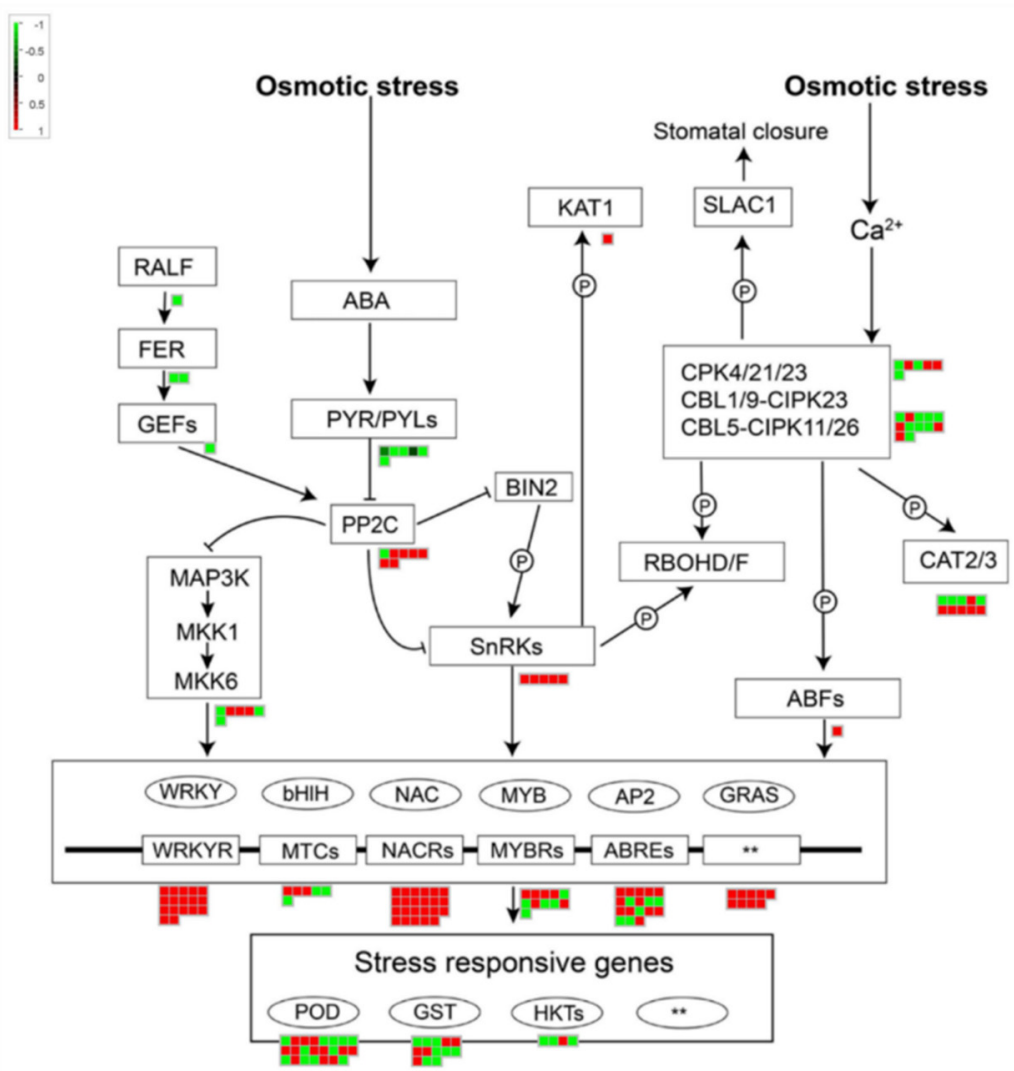

Figure 5. Genes involved in response to salt and drought stress in I. lactea var. chinensis transcriptomes. Relative expression profiles were showed in green-black-red scale, red indicates $\log _{2}$ (fold change) $>0$, green indicates $\log _{2}$ (fold change) $<0$. ABA, abscisic acid; PYR/PYLs, pyrabactin resistance 1-like protein; PP2C, type 2C protein phosphatases; SnRKs, Snf1 (sucrose non-fermenting-1)-related protein kinases; BIN2, GSK3-like kinase; RBOHD/F, respiratory burst oxidase homolog D; KAT, $\mathrm{K}^{+}$Channel; SLAC1, slow anion channel 1; CPK/CBL-CIPK, calcium-regulated phosphorylation systems; CAT2/3, catalase 2/3; RALF, rapid alkalinization factor; FER, Feronia; GEF, guanine nucleotide exchange factor; MAPK, mitogen-activated protein kinase; $A B F s, A B F$, transcription factor; WRKY, WRKY transcription factor; $\mathrm{bHlH}, \mathrm{bHlH}$ transcription factor; NAC, NAC transcription factor; MYB, MYB transcription factor; AP2, AP2/ERF transcription factor, GRAS, GRAS transcription factor; POD, peroxidase; GST, glutathione S-transferase zeta; HKTs, cation transporters. Adapted with permission from Chen et al. [31]. Copyright 2021 Wiley Online Library, Zhu et al. [3] Copyright 2016 Elsevier, Luo et al. [1] Copyright 2019 Springer Nature. “**” indicate unknown cis-elements.

The results showed that a total of 22 DEGs were mapped to the ABA signaling pathway, of which six DEGs were mapped to the ABA receptor PYRs/PYL, and all of them were in a downward trend; five DEGs were mapped to the threonine/serine receptor kinase $S n R K 2$, and all of them were in an upward trend; seven DEGs were mapped to the ABA coreceptor $P P 2 C$, most of them were in an upward trend; and one DEG mapped to rapid alkalinization factor 1 (RALF), two mapped to receptor-like kinase FERONIA (FER), and 1 mapped to the guanine nuclear exchange factor (GEF) were all in a downward trend.

A total of 35 DEGs were mapped to the osmotic stress signaling pathway, among which $M A P K s$ were mapped to six and three were upregulated; secondary messengers $C P K$ s and $C B L s-C I P K$ s were mapped to 18 and 7 were upregulated; potassium channel (KAT1) was mapped to one DEG, which was upregulated.

In addition, 85 DEGs were mapped as TFs. Among them, the expression of $1 A B F$ was upregulated; $17 \mathrm{WRKYs}$ were all upregulated; three of six $b H l H$ s were upregulated; 
23 NACs were all upregulated; 6 of 11 MYBs were upregulated; 12 of 18 AP2/ERFs were upregulated; and nine GRASs were all upregulated. It is worth noting that $P O D, C A T$, and GST related to non-enzymatic antioxidants have also been mapped to 23,10 , and 13, respectively (Table S6).

\section{Discussion}

I. lactea var. chinensis is a perennial halophyte. Due to its salt tolerance, drought tolerance, and high ornamental value, I. lactea var. chinensis has become an important plant for improving saline-alkali land [32,33]. In recent years, our research team has used second-generation sequencing technology to identify genes in I. lactea var. chinensis related to cadmium and salt stress $[10,11]$, but we did not conduct a comparison of regulatory mechanisms under drought and salt stress. In addition, according to our previous research, I. lactea var. chinensis is most sensitive under $1.5 \% \mathrm{NaCl}$ stress [34]. This concentration may cause salt shock in plants and trigger the expression of salt shock-related genes [35]. Therefore, in our study, $1.5 \% \mathrm{NaCl}$ was used as the treatment concentration to explore the gene expression of I. lactea var. chinensis under salt shock, and to compare with the treatment of $30 \%$ PEG, so as to explore the common regulatory mechanism under salt and drought stress.

Compared with second-generation sequencing technology, SMRT sequencing technology can generate full-length transcripts due to its long reading length. This can greatly improve the accuracy of transcriptome data characterization, which plays an important role in the research of important functional genes in plants [36]. In this study, we used the PacBio RSII platform to analyze expression patterns under $\mathrm{NaCl}$ and PEG stress for the first time. The average length of the transcripts using the PacBio RS II platform was $1564 \mathrm{bp}$, which was 1.97 times higher than the average length of the 793 NGS platform [10]. Overall, $28,419(91.10 \%)$ full-length transcripts were annotated at least once by seven databases, among which 12,556 were annotated in KEGG, 22,089 in COG, 28,063 in NR, 21,697 in GO, and the remaining non-annotated transcripts may represent specific genes. The results will expand the pool of tools available for potential gene exploration. We also used the Illumina platform to analyze the gene expression under $\mathrm{NaCl}$ and PEG stress. A total of 62,718 transcripts were identified in nine sample libraries, with more than 20,000 genes in each library. This indicated that the expression of most genes was relatively stable, and the specific expression of genes during abiotic stress was lower. We also found that 3863 DEGs were up-regulated or down-regulated under both $\mathrm{NaCl}$ and PEG stress, which indicated that these genes were related to both salt tolerance and drought resistance. These genes will become the focus of future research.

To identify the key genes in response to drought and salt stress further, we carried out WGCNA analysis with all DEGs and selected 10 key genes for salt and drought stress, respectively. According to the results of the NCBI blast, these genes play important roles in plant response to salt stress. For example, AtOSCA1 in Arabidopsis thaliana (A. thaliana) could act as a $\mathrm{Ca}^{2+}$ permeable channel, which played a key role in the initial stage of the hypertonic stress response [37]. The PP2C-a10 gene of the PP2C family in wheat could improve the drought resistance of transgenic A. thaliana [38]. Ectopic expression of the TMKP1 protein of the MAPK family in wheat and the Arabidopsis mkp1 mutant could improve salt stress tolerance [39]. The overexpression of the OsCPK21 gene could significantly improve the salt tolerance of rice [40], the overexpression of the PYL5 gene in rice can improve tolerance to drought and salt stress [41], and the overexpression of the accbl1 gene in the pineapple could significantly improve tolerance to drought and salt stress [42]. According to the analysis results of these genes in other species, we found that these key genes may also play an important role in response to drought and salt stress in $I$. lactea var. chinensis, and will also be included in follow-up stress research.

Drought and salt can induce osmotic stress in plants, and then secondary messengers are synthesized [43]. The changes in secondary messengers act as signals to activate different signal cascades. They are the main regulators of signal transduction, which can 
effectively link external stimuli with cell responses and thus activate the expression of downstream functional genes [3]. In this study, we found 35 DEGs related to osmotic stress signal transduction in DEGs under drought and salt stress, including 6 CDPKs, 12 CIPKs, and 6 MAPKs. Among them, three CDPK-related genes, four CIPK-related genes, and three MAPK-related genes were upregulated, which indicated that the genes related to osmotic stress in I. lactea var. chinensis would be highly activated within $24 \mathrm{~h}$. Through GO enrichment analysis, it was found that transporter activity, transmembrane transporter activity, and membrane, which are closely related to osmotic stress, were significantly enriched under the two stresses. In addition, 3863 DEGs with the same expression pattern under the two stresses were also significantly enriched in oxidoreductase activity, transmembrane transporter activity, and transmembrane. At the same time, DEGs with the same expression pattern were also enriched in amino acid metabolism, carbohydrate metabolism, signaling and cellular processes, ion channels, and other KEGG pathways, which suggests that there is an obvious co-regulatory system between $\mathrm{NaCl}$ and PEG induced osmotic response pathways. These findings are similar to those previously observed in Arabidopsis [44], alfalfa [1], rice [45], and red bean [13]. These results showed that when I. lactea var. chinensis was challenged by drought and salt stress, osmotic stress would occur. At this time, amino acid metabolism, antioxidant enzymes, and other defense systems would be activated through ion channels and signal transduction systems, thus playing a role in response to stress.

The ABA signal transduction pathway is also the core of plant response to abiotic stress. When plants detect abiotic stress, they activate the SnRK2 receptor through $\mathrm{Ca}^{2+}$ signaling, thus stimulating the production of ABA. The PYL receptor then binds to ABA and prevents PP2C-mediated dephosphorylation of SnRK2, resulting in SnRK2 kinase activation. When SnRK2 is activated, it can phosphorylate downstream TFs and activate the expression of ABA-dependent genes, resulting in an ABA response [3]. In this study, we found 22 DEGs related to ABA signal transduction in common DEGs, including six PYL, seven $P P 2 C$, and five SnRK2 genes. The transcripts of the six PYLs were downregulated, while the other genes, including seven $P P 2 C$ and five $S n R K 2$, were upregulated after stress. These results were consistent with known ABA regulatory pathways [1,46], implying that abiotic stress may cause the activation of the ABA signaling pathway and affect the expression of PYL, PP2C, and SnRK2 genes. PYR/PYLs were downregulated under salt and drought treatment, while the $P P 2 C$ in the same pathway was induced accordingly.

TFs are important parts of the abiotic stress-mediated signaling pathway, which are phosphorylated by protein kinases, and directly regulate the expression of downstream stress response genes [47]. The GRAS TF family is related to various biological processes, such as root and meristem development, light signal transduction, and biological and abiotic stress responses [48]. Under the two stresses in this study, nine GRAS-related DEGs were co-expressed, and all of them had a trend of upregulated expression, which indicated that I. lactea var. chinensis could reduce the damage caused by drought and salt stress by regulating the expression of GRAS. The MYB family is another type of TF involved in plant responses to environmental stress [49]. Genetic analysis showed that the TaMYBsm3 gene was overexpressed in wheat, which improved drought tolerance, increased proline content, and decreased MDA content [50]. In this study, 11 MYB-related DEGs were found to be expressed under two stresses, of which six were upregulated, and five were down-regulated. Different expression patterns indicated that the MYB TF family played multiple roles in response to stress. In addition, the NAC and WRKY TF families also play a very important role in the plant response to stress. For example, NAC transcription factor JUNGBRUNNEN1 in tomato increased drought tolerance [51], and the WRKY TF GmWRKY12 endowed soybean with drought and salt tolerance [52]. In the two types of DEGs, 23 NACs and 17 WRKYs were identified, and all of them were upregulated, which indicated that $I$. lactea var. chinensis could protect against drought and salt stress by up-regulating NAC and WRKY genes. BHLH and AP2/ERF are also important families of TFs in plant response to stress [53,54]. In this study, 6 bHLHs and $18 \mathrm{AP} 2 /$ ERF-related DEGs 
were found to participate in response to drought and salt stress and showed inducible and inhibitory expression patterns, which indicated that complex transcriptional regulation could be involved in the adaptation of I. lactea var. chinensis to adverse environments.

Under abiotic stress, a large number of ROS are produced, leading to cell oxidative damage and death. Plants will activate different defense/metabolism pathways to remove and maintain the level of ROS, thus protecting cells from oxidative damage [55]. Previous studies found that $130 \mathrm{DEGs}$ related to active oxygen scavenging were activated in I. lactea var. chinensis under salt stress, which indicated that I. lactea var. chinensis could maintain the ROS in cells at a relatively low level by activating an antioxidant defense system [11]. In this study, DEGs shared by I. lactea var. chinensis under the two stresses were also significantly enriched in GO terms, such as oxidoreductase activity, and KEGG pathways, such as alanine, aspartate, and glutamate metabolism, ascorbate and aldarate metabolism, which indicated that $\mathrm{NaCl}$ and PEG stress also activated the antioxidant defense system at the molecular level. In addition, antioxidant enzyme-related DEGs (e.g., POD, CAT) and non-enzyme antioxidant-related DEGs (e.g., GST) were also significantly regulated by the two stresses, which was consistent with previous reports on I. lactea var. chinensis under salt stress [11]. These results indicate that these DEGs play an important role in response to osmotic stress, thus protecting plants from ROS damage.

\section{Conclusions}

In this study, we sequenced the full-length transcriptome of I. lactea var. chinensis and analyzed the differential gene expression patterns under $\mathrm{NaCl}$ and PEG stress by second-generation sequencing technology. Through WGCNA analysis, key genes were screened in response to stress. Moreover, genes with the same expression pattern were the focus of analysis. Plants are often affected by multiple abiotic stresses. We hope that our research can provide some reference for the cross-study of salt and drought tolerance.

Supplementary Materials: The following are available online at https:/ /www.mdpi.com/2073-442 5/12/3/434/s1, Table S1: BUSCO analysis of the full-length transcripts, Table S2: BLAST analysis of the full-length transcripts against public databases, Table S3: The mapping between the Illumina reads of each sample and the reference transcript sequences generated by PacBio Iso-Seq, Table S4: The Hub gene under $\mathrm{NaCl}$ stress, Table S5: The Hub gene under PEG stress, Table S6: DEGs involved in stress defense under $\mathrm{NaCl}$ and PEG stress.

Author Contributions: Conceptualization, C.G.; Methodology, C.G.; Investigation, C.G., L.N., Z.W., J.G., and X.P.; Resources, L.L., H.L., and H.Y.; Data curation, C.G., L.N., and Z.W.; Writing-original draft preparation, C.G., L.N., and Z.W.; Writing-review and editing, C.G. and L.N. All authors have read and agreed to the published version of the manuscript.

Funding: This research was funded by the National Natural Science Foundation of China (31772351) and 333 Talents Project of Jiangsu Province (BRA2017498).

Institutional Review Board Statement: Not applicable.

Informed Consent Statement: Not applicable.

Data Availability Statement: The data have been deposited to the National Center for Biotechnology Information (NCBI) under accession number PRJNA697945.

Conflicts of Interest: The authors declare no conflict of interest.

\section{References}

1. Luo, D.; Zhou, Q.; Wu, Y.; Chai, X.; Liu, W.; Wang, Y.; Yang, Q.; Wang, Z.; Liu, Z. Full-length transcript sequencing and comparative transcriptomic analysis to evaluate the contribution of osmotic and ionic stress components towards salinity tolerance in the roots of cultivated alfalfa (Medicago sativa L.). BMC Plant Biol. 2019, 19. [CrossRef] [PubMed]

2. Chaves, M.M.; Flexas, J.; Pinheiro, C. Photosynthesis under drought and salt stress: Regulation mechanisms from whole plant to cell. Ann. Bot. 2009, 103, 551-560. [CrossRef] [PubMed]

3. Zhu, J.-K. Abiotic Stress Signaling and Responses in Plants. Cell 2016, 167, 313-324. [CrossRef] 
4. Cutler, S.R.; Rodriguez, P.L.; Finkelstein, R.R.; Abrams, S.R. Abscisic acid: Emergence of a core signaling network. Annu. Rev. Plant Biol. 2010, 61, 651-679. [CrossRef] [PubMed]

5. Choudhury, F.K.; Rivero, R.M.; Blumwald, E.; Mittler, R. Reactive oxygen species, abiotic stress and stress combination. Plant J. 2017, 90, 856-867. [CrossRef] [PubMed]

6. Zhu, J.-K. Salt and drought stress signal transduction in plants. Annu. Rev. Plant Biol. 2002, 53, 247-273. [CrossRef] [PubMed]

7. Zhu, J.-K. Genetic analysis of plant salt tolerance using Arabidopsis: Figure 1. Plant Physiol. 2000, 124, 941-948. [CrossRef] [PubMed]

8. Zhao, C.; Zhang, H.; Song, C.; Zhu, J.-K.; Shabala, S. Mechanisms of plant responses and adaptation to soil salinity. Innovation 2020, 1, 100017. [CrossRef]

9. Jiang, Z.; Zhou, X.; Tao, M.; Yuan, F.; Liu, L.; Wu, F.; Wu, X.; Xiang, Y.; Niu, Y.; Liu, F.; et al. Plant cell-surface GIPC sphingolipids sense salt to trigger $\mathrm{Ca}^{2+}$ influx. Nature 2019, 572, 341-346. [CrossRef] [PubMed]

10. Gu, C.-S.; Liu, L.-Q.; Deng, Y.-M.; Zhang, Y.-X.; Wang, Z.-Q.; Yuan, H.-Y.; Huang, S.-Z. De novo characterization of the Iris lactea var. chinensis transcriptome and an analysis of genes under cadmium or lead exposure. Ecotoxicol. Environ. Saf. 2017, 144, 507-513. [CrossRef]

11. Gu, C.; Xu, S.; Wang, Z.; Liu, L.; Zhang, Y.; Deng, Y.; Huang, S. De novo sequencing, assembly, and analysis of Iris lactea var. chinensis roots' transcriptome in response to salt stress. Plant Physiol. Biochem. 2018, 125. [CrossRef] [PubMed]

12. Liang, Y.; Chen, S.-Y.; Liu, G.-S. Application of next generation sequencing techniques in plant transcriptome. YiChuan 2011, 33, 1317-1326.

13. Zhu, Z.; Chen, H.; Xie, K.; Liu, C.; Li, L.; Liu, L.; Han, X.; Jiao, C.; Wan, Z.; Sha, A. Characterization of drought-responsive transcriptome during seed germination in Adzuki bean (L.) by PacBio SMRT and Illumina Sequencing. Front. Genet. 2020, 11, 996. [CrossRef]

14. Sun, M.; Huang, D.; Zhang, A.; Khan, I.; Yan, H.; Wang, X.; Zhang, X.; Zhang, J.; Huang, L. Transcriptome analysis of heat stress and drought stress in pearl millet based on Pacbio full-length transcriptome sequencing. BMC Plant Biol. 2020, 20. [CrossRef]

15. Zhang, G.; Sun, M.; Wang, J.; Lei, M.; Li, C.; Zhao, D.; Huang, J.; Li, W.; Li, S.; Li, J.; et al. PacBio full-length cDNA sequencing integrated with RNA-seq reads drastically improves the discovery of splicing transcripts in rice. Plant J. 2019, 97, 296-305. [CrossRef] [PubMed]

16. Zhang, D.; Li, W.; Chen, Z.-J.; Wei, F.-G.; Liu, Y.-L.; Gao, L.-Z. SMRT- and Illumina-based RNA-seq analyses unveil the ginsinoside biosynthesis and transcriptomic complexity in Panax notoginseng. Sci. Rep. 2020, 10, 15310. [CrossRef]

17. Fu, L.; Niu, B.; Zhu, Z.; Wu, S.; Li, W. CD-HIT: Accelerated for clustering the next-generation sequencing data. Bioinformatics 2012, 28, 3150-3152. [CrossRef]

18. Simão, F.A.; Waterhouse, R.M.; Ioannidis, P.; Kriventseva, E.V.; Zdobnov, E.M. BUSCO: Assessing genome assembly and annotation completeness with single-copy orthologs. Bioinformatics 2015, 31, 3210-3212. [CrossRef]

19. Wagner, G.P.; Kin, K.; Lynch, V.J. Measurement of mRNA abundance using RNA-seq data: RPKM measure is inconsistent among samples. Theory Biosci. 2012, 131, 281-285. [CrossRef] [PubMed]

20. Robinson, M.D.; McCarthy, D.J.; Smyth, G.K. edgeR: A Bioconductor package for differential expression analysis of digital gene expression data. Bioinformatics 2010, 26, 139-140. [CrossRef]

21. Schurch, N.J.; Schofield, P.; Gierliński, M.; Cole, C.; Sherstnev, A.; Singh, V.; Wrobel, N.; Gharbi, K.; Simpson, G.G.; Owen-Hughes, T.; et al. How many biological replicates are needed in an RNA-seq experiment and which differential expression tool should you use? RNA 2016, 22, 839-851. [CrossRef] [PubMed]

22. Love, M.I.; Huber, W.; Anders, S. Moderated estimation of fold change and dispersion for RNA-seq data with DESeq2. Genome Biol. 2014, 15, 550. [CrossRef]

23. Young, M.D.; Wakefield, M.J.; Smyth, G.K.; Oshlack, A. Gene ontology analysis for RNA-seq: Accounting for selection bias. Genome Biol. 2010, 11, R14. [CrossRef]

24. Mao, X.; Cai, T.; Olyarchuk, J.G.; Wei, L. Automated genome annotation and pathway identification using the KEGG Orthology (KO) as a controlled vocabulary. Bioinformatics 2005, 21, 3787-3793. [CrossRef]

25. Chen, C.; Chen, H.; Zhang, Y.; Thomas, H.R.; Frank, M.H.; He, Y.; Xia, R. TBtools: An Integrative Toolkit Developed for Interactive Analyses of Big Biological Data. Mol. Plant 2020, 13, 1194-1202. [CrossRef]

26. Ramsak, Ž.; Baebler, Š.; Rotter, A.; Korbar, M.; Mozetic, I.; Usadel, B.; Gruden, K. GoMapMan: Integration, consolidation and visualization of plant gene annotations within the MapMan ontology. Nucleic Acids Res. 2014, 42, D1167-D1175. [CrossRef] [PubMed]

27. Langfelder, P.; Horvath, S. WGCNA: An R package for weighted correlation network analysis. BMC Bioinform. 2008, 9, 559. [CrossRef] [PubMed]

28. Shannon, P.; Markiel, A.; Ozier, O.; Baliga, N.S.; Wang, J.T.; Ramage, D.; Amin, N.; Schwikowski, B.; Ideker, T. Cytoscape: A software environment for integrated models of biomolecular interaction networks. Genome Res. 2003, 13, 2498-2504. [CrossRef] [PubMed]

29. Chin, C.-H.; Chen, S.-H.; Wu, H.-H.; Ho, C.-W.; Ko, M.-T.; Lin, C.-Y. cytoHubba: Identifying hub objects and sub-networks from complex interactome. BMC Syst. Biol. 2014, 8, S11. [CrossRef] [PubMed]

30. Furuno, M.; Kasukawa, T.; Saito, R.; Adachi, J.; Suzuki, H.; Baldarelli, R.; Hayashizaki, Y.; Okazaki, Y. CDS annotation in full-length cDNA sequence. Genome Res. 2003, 13, 1478-1487. [CrossRef] 
31. Chen, X.; Ding, Y.; Yang, Y.; Song, C.; Wang, B.; Yang, S.; Guo, Y.; Gong, Z. Protein kinases in plant responses to drought, salt, and cold stress. J. Integr. Plant Biol. 2021. [CrossRef]

32. Zhang, B.; Li, P.-F.; Fan, F.-C. Ionic relations and proline accumulation in shoots of two Chinese Iris germplasms during NaCl stress and subsequent relief. Plant Growth Regul. 2012, 68, 49-56. [CrossRef]

33. Tang, J.; Liu, Q.; Yuan, H.; Zhang, Y.; Wang, W.; Huang, S. Molecular cloning and characterization of a novel salt-specific responsive WRKY transcription factor gene IlWRKY2 from the halophyte Iris lactea var. chinensis. Genes Genom. 2018, 40, 893-903. [CrossRef] [PubMed]

34. Tong, H.Y.; Gu, C.S.; Yuan, H.Y.; Ma, J.J.; Huang, S.Z. Effects of salt stress on the growth, photosynthetic pigments and structure of two halophytes, Iris halophila and I. lactea var. chinensis. Fresenius Environ. Bull. 2014, 23, 84-90.

35. Shavrukov, Y. Salt stress or salt shock: Which genes are we studying? J. Exp. Bot. 2013, 64, 119-127. [CrossRef] [PubMed]

36. Sun, S.; Li, Y.; Chu, L.; Kuang, X.; Song, J.; Sun, C. Full-length sequencing of ginkgo transcriptomes for an in-depth understanding of flavonoid and terpenoid trilactone biosynthesis. Gene 2020, 758, 144961. [CrossRef]

37. Liu, X.; Wang, J.; Sun, L. Structure of the hyperosmolality-gated calcium-permeable channel OSCA1.2. Nat. Commun. 2018, 9, 5060. [CrossRef]

38. Yu, X.; Han, J.; Li, L.; Zhang, Q.; Yang, G.; He, G. Wheat PP2C-a10 regulates seed germination and drought tolerance in transgenic Arabidopsis. Plant Cell Rep. 2020, 39, 635-651. [CrossRef] [PubMed]

39. Zaidi, I.; Ebel, C.; Belgaroui, N.; Ghorbel, M.; Amara, I.; Hanin, M. The wheat MAP kinase phosphatase 1 alleviates salt stress and increases antioxidant activities in Arabidopsis. J. Plant Physiol. 2016, 193, 12-21. [CrossRef]

40. Asano, T.; Hakata, M.; Nakamura, H.; Aoki, N.; Komatsu, S.; Ichikawa, H.; Hirochika, H.; Ohsugi, R. Functional characterisation of OsCPK21, a calcium-dependent protein kinase that confers salt tolerance in rice. Plant Mol. Biol. 2011, 75, 179-191. [CrossRef] [PubMed]

41. Kim, H.; Lee, K.; Hwang, H.; Bhatnagar, N.; Kim, D.-Y.; Yoon, I.S.; Byun, M.-O.; Kim, S.T.; Jung, K.-H.; Kim, B.-G. Overexpression of PYL5 in rice enhances drought tolerance, inhibits growth, and modulates gene expression. J. Exp. Bot. 2014, 65, 453-464. [CrossRef]

42. Aslam, M.; Fakher, B.; Jakada, B.H.; Zhao, L.; Cao, S.; Cheng, Y.; Qin, Y. Genome-wide identification and expression profiling of CBL-CIPK gene family in Pineapple and the role of CBL1 in abiotic and biotic stress response. Biomolecules 2019, 9, 293. [CrossRef] [PubMed]

43. Wu, Y.; Wei, W.; Pang, X.; Wang, X.; Zhang, H.; Dong, B.; Xing, Y.; Li, X.; Wang, M. Comparative transcriptome profiling of a desert evergreen shrub, Ammopiptanthus mongolicus, in response to drought and cold stresses. BMC Genom. 2014, 15, 671. [CrossRef]

44. Wong, C.E.; Li, Y.; Labbe, A.; Guevara, D.; Nuin, P.; Whitty, B.; Diaz, C.; Golding, G.B.; Gray, G.R.; Weretilnyk, E.A.; et al. Transcriptional profiling implicates novel interactions between abiotic stress and hormonal responses in Thellungiella, a close relative of Arabidopsis. Plant Physiol. 2006, 140, 1437-1450. [CrossRef]

45. Rabbani, M.A.; Maruyama, K.; Abe, H.; Khan, M.A.; Katsura, K.; Ito, Y.; Yoshiwara, K.; Seki, M.; Shinozaki, K.; YamaguchiShinozaki, K. Monitoring expression profiles of rice genes under cold, drought, and high-salinity stresses and abscisic acid application using cDNA microarray and RNA gel-blot analyses. Plant Physiol. 2003, 133, 1755-1767. [CrossRef]

46. Chan, Z. Expression profiling of ABA pathway transcripts indicates crosstalk between abiotic and biotic stress responses in Arabidopsis. Genomics 2012, 100, 110-115. [CrossRef] [PubMed]

47. Huang, G.-T.; Ma, S.-L.; Bai, L.-P.; Zhang, L.; Ma, H.; Jia, P.; Liu, J.; Zhong, M.; Guo, Z.-F. Signal transduction during cold, salt, and drought stresses in plants. Mol. Biol. Rep. 2012, 39, 969-987. [CrossRef]

48. Zhang, S.; Li, X.; Fan, S.; Zhou, L.; Wang, Y. Overexpression of HcSCL13, a Halostachys caspica GRAS transcription factor, enhances plant growth and salt stress tolerance in transgenic Arabidopsis. Plant Physiol. Biochem. 2020, 151, 243-254. [CrossRef] [PubMed]

49. Li, J.; Han, G.; Sun, C.; Sui, N. Research advances of MYB transcription factors in plant stress resistance and breeding. Plant Signal Behav. 2019, 14, 1613131. [CrossRef]

50. Li, Y.; Zhang, S.; Zhang, N.; Zhang, W.; Li, M.; Liu, B.; Shi, Z. MYB-CC transcription factor, TaMYBsm3, cloned from wheat is involved in drought tolerance. BMC Plant Biol. 2019, 19, 143. [CrossRef] [PubMed]

51. Thirumalaikumar, V.P.; Devkar, V.; Mehterov, N.; Ali, S.; Ozgur, R.; Turkan, I.; Mueller-Roeber, B.; Balazadeh, S. NAC transcription factor JUNGBRUNNEN1 enhances drought tolerance in tomato. Plant Biotechnol. J. 2018, 16, 354-366. [CrossRef] [PubMed]

52. Shi, W.-Y.; Du, Y.-T.; Ma, J.; Min, D.-H.; Jin, L.-G.; Chen, J.; Chen, M.; Zhou, Y.-B.; Ma, Y.-Z.; Xu, Z.-S.; et al. The WRKY Transcription Factor GmWRKY12 Confers Drought and Salt Tolerance in Soybean. Int. J. Mol. Sci. 2018, 19, 4087. [CrossRef] [PubMed]

53. Sun, X.; Wang, Y.; Sui, N. Transcriptional regulation of bHLH during plant response to stress. Biochem. Biophys. Res. Commun. 2018, 503, 397-401. [CrossRef]

54. Xie, Z.; Nolan, T.M.; Jiang, H.; Yin, Y. AP2/ERF transcription factor regulatory networks in hormone and abiotic stress Responses. Front. Plant Sci. 2019, 10, 228. [CrossRef] [PubMed]

55. Golldack, D.; Li, C.; Mohan, H.; Probst, N. Tolerance to drought and salt stress in plants: Unraveling the signaling networks. Front. Plant Sci. 2014, 5, 151. [CrossRef] 